\title{
What Can Tone and Entropy Tell Us About Risk of Cardiovascular Diseases?
}

\author{
Ahsan H Khandoker ${ }^{1}$, Yahya Al Zaabi ${ }^{1}$, Herbert F Jelinek ${ }^{2}$ \\ ${ }^{1}$ Healthcare Engineering Innovation Center (HEIC), Khalifa University, Abu Dhabi, United Arab Emirates \\ ${ }^{2}$ Charles Sturt University, Albury, Australia
}

\begin{abstract}
A validated invasive method to calculate cardiovascular risk is using the Framingham cardiovascular risk score. Causes of CVD include atherosclerosis. The level of atherosclerosis is measured by the total cholesterol to high-density lipoprotein (TC/HDL) ratio. Causes of rhythm disturbance include both intrinsic and extrinsic factors. QT interval dispersion (QTd) indicates ventricular repolarization. The autonomic nervous system can play an essential role in the functional and pathological of the cardiovascular system. Tone and entropy are measures of the mean sympathovagal balance and the total autonomic nervous system activity influencing heart rate. The autonomic nervous system can also provide information on the risk of CVD presence. Tone-entropy analysis indicated that tone is associated to both TC/HDL and QTD length for mild and very high risk group. Entropy was not associated with both TC/HDL and QTD length in any risk categories. Our findings suggest that this may be of use for initial screening for CVD risk as it is noninvasive and easy to perform method.
\end{abstract}

\section{Introduction}

Cardiovascular disease (CVD) is the leading cause of mortality and morbidity in the world. Multifactorial factors are involved in increasing the risk of CVD including cholesterol level, gender, age, diabetes and systolic blood pressure with atherosclerosis manifesting as an early indicator of the disease [1,2]. The dysfunction of autonomic nervous system (ANS) called as cardia autonomic neuropathy (CAN) has a negative impact on the functional of the cardiovascular system [3].

The development and progression of atherosclerosis is related to the level of cholesterol and specifically lowdensity lipoprotein cholesterol (LDL-C). LDL-C can be damaged by chemical interactions with free radicals to produce an oxidized version of LDL-C. This can result in hardening arteries leading to atherosclerosis, CVD and CAN [4,5]. Thus, a link between cholesterol level and autonomic nervous system function is of interest for early identification of increased risk associated with coronary artery disease complicated by CAN and increased risk of sudden cardiac death [6].
To decrease the risk of sudden cardiac death, noninvasive testing for the risk and presence of CVD has become a critical area in clinical research. Changes in traditional ECG markers such as ST segments, QRS, Pwave changes have been documented and clinically used to test for cardiac abnormalities. QT interval dispersion (QTD) on the surface of ECG measures ventricular repolarization. Increased QTD in surface of ECG has been linked to increased inhomogeneity of ventricular repolarization [7]. Beside that the ECG morphologies can be represented by QTD measures, the ECG also demonstrates the contributions of the automimic system in regulation of heart rhythm. Evidence that QTD, to certain extent, is due to automimic regulation has been reported in [8].

The autonomic nervous system (ANS) can also be viewed as a modulator that regulates interbeat interval variation. Tone and entropy are measures of the mean sympathovagal balance and the total autonomic nervous system activity influencing heart rate. Tone-entropy (T_E) algorithm has also been reported to differentiate between diabetes with and without CAN [9].

One validated method to calculate cardiovascular risk is using the Framingham cardiovascular risk score (FRS). This equation uses data on age, gender, cholesterol level, blood pressure, smoking and diabetes status to provide a risk score from mild to very high CVD risk over 10 years $[10,11]$. Assessment of ANS can also provide information on the risk of CVD presences. As such cholesterol level, one component of FRS, is a function of the ANS [12]. The aim of this study is to investigate the relationship between Framingham CVD risk score with TC/HDL and QT interval dispersion using T-E algorithm.

\section{Method}

\subsection{Participants}

Data was collected from 230 subjects at the Charles Sturt University Diabetes Health (Diab Health) Clinic. Inclusion in the analysis required a complete dataset for the patient including tone and entropy parameters as well as age, sex, BGL, blood pressure, cholesterol profile, and BMI. Exclusion criteria from the study were presence of 
cardiovascular, including peripheral vascular or cerebrovascular disease, and signs or symptoms suggestive of arrhythmia such as palpitation, hypotension, syncope or chest pain. Cholesterol profile was determined by South West Pathology in Albury. The normal ranges are TC 3.9$3.5 \mathrm{mmol} / \mathrm{L}$, Triglycerides $0.5-1.7 \mathrm{mmol} / \mathrm{L}$, HDL $0.8-$ $1.5 \mathrm{mmol} / \mathrm{L}$, LDL $1.7-3.5 \mathrm{mmol} / \mathrm{L}$ and TC/HDL $<5$. The cardiac risk score was determined by the following variables: age, gender, cholesterol , blood pressure, diabetes status and smoking and was divided into four groups: very high $(>20 \%)$, high $(15-20 \%)$, moderate $(10-$ $15 \%$ ) and mild (less than 10\%) as defined by the Framingham risk score.

\subsection{ECG recording}

RR intervals were determined from 12-lead ECG recordings obtained using the CardioPerfect workstation (Welsh-Allyn, Netherlands).

\subsection{Tone-Entropy (T-E) Determination}

The T-E algorithm was previously described (15-17). Heart period data, RR intervals are transformed into percentage index (PI) time series:

$$
P I(n)=[H(n)-H(n+1)] \times 100 / H(n)
$$

where $[H(n)]$ is a heart period time series, and $n$ a serial number of heart beats. The tone is defined as a first order moment (arithmetic average) of this PI time series as:

$$
\sum_{n} P I(n) / N \quad \text { (non-dimensional) }
$$

where $\mathrm{N}$ is a total number of PI terms. The tone, which represents the balance between accelerations $(\mathrm{PI}>0)$ and inhibitions $(\mathrm{PI}<0)$ of the heart, represents the sympathovagal balance (18). The entropy is defined on PI probability distribution by using Shannon's formula:

$$
-\sum_{n} p(i) \log _{2} p(i)
$$

where $[p(i)]$ is a probability that $\operatorname{PI}(\mathrm{n})$ has a value in the range, $i<\operatorname{PI}(n)<i+1, i$ an integer. The entropy evaluates total acceleration-inhibition activities, or total heart period variations.

\subsection{Statistical Analysis}

Results were expressed as means $( \pm \mathrm{SD})$. One-way analysis of variance (ANOVA) and Bonferroni post hoc were used for comparison among the four Framingham risk groups (mild, moderate, high, and very high) to evaluate whether statistically significant difference exist among the risk groups. Kruskal Wallis statistics were used to determine the range for which TC/HDL or QTD stratified CVD risk groups into two distributions for tone and entropy. The obtained $p$ value provides the point or range of influence and was set at $p<0.05$. The smaller the point of influence is the greater the interaction between TC/HDL or QTD and tone or entropy.

\section{Results}

Data from 230 participants was analysed for a possible relationship between Framingham risk groups using the tone-entropy (T-E) algorithm and cholesterol level measured as TC/HDL and QT interval dispersion (QTD).

Dividing the participants into four groups: very high (>20\%), high (15-20\%), moderate (10-15\%) and mild (less than $10 \%$ ) according to the Framingham risk score model showed that the very high risk group had a higher risk

\begin{tabular}{|c|c|c|c|c|c|}
\hline & Mild & Moderate & High & Very high & $\mathbf{P}$ \\
\hline Participants & 137 & 87 & 25 & 24 & - \\
\hline Age (yrs) & $61.22 \pm 10.5$ & $66.82 \pm 8.7$ & $69.83 \pm 8.8$ & $72.17 \pm 6.4$ * & $<0.0001$ \\
\hline DM, yes(no) & $8(129)$ & 11(33) & $14(11)$ & $16(8)$ & - \\
\hline $\operatorname{Sex} M(F)$ & $41(96)$ & $16(28)$ & 19(6) & 18(6) & - \\
\hline WC (cm) & $92.36 \pm 14.1$ & $95.41 \pm 12.1$ & $100.14 \pm 13.9$ & $101.75 \pm 13.4$ & 0.060 \\
\hline BMI & $27.28 \pm 5.7$ & $27.93 \pm 4.4$ & $26.82 \pm 7.1$ & $29.58 \pm 5.9$ & 0.309 \\
\hline $\begin{array}{l}\text { SBP } \\
(\mathrm{mmHg})\end{array}$ & $122.59 \pm 13.1$ & $143.32 \pm 19.0$ & $140.33 \pm 15.9$ & $149.22 \pm 17.8^{*}$ & $<0.0001$ \\
\hline $\begin{array}{l}\text { DBP } \\
\text { (mmHg) }\end{array}$ & $74.20 \pm 7.8$ & $78.89 \pm 10.8$ & $78.58 \pm 12.4$ & $78.52 \pm 12.7$ & 0.0114 \\
\hline $\begin{array}{l}\text { BGL } \\
(\mathrm{mmol} / \mathrm{L})\end{array}$ & $5.07 \pm 0.9$ & $5.56 \pm 1.2$ & $6.08 \pm 1.2$ & $6.85 \pm 3.4^{*}$ & $<0.0001$ \\
\hline HbA1c & $5.78 \pm 0.5$ & $6.14 \pm 0.7$ & $6.19 \pm 0.8$ & $6.34 \pm 1.3^{*}$ & 0.0007 \\
\hline
\end{tabular}
profile for atherosclerosis with significantly higher waist circumference, BMI, BGL and HbAlc results (Table 1)

$\overline{\mathrm{DM}}$ - diabetes mellitus; WC - waist circumference, BMI - body mass index; SBP - systolic blood pressure, DBP - diastolic blood pressure; BGL - blood glucose level; HbA1c - glycosylated haemoglobin, ${ }^{*} \mathrm{p}<0.01$ for Mild vs. Very high.

The cholesterol profile for participants divided into four risk groups is shown in Table 2 . Only HDL is significantly different. In all cases, the very high risk groups had lower levels possibly due to medication use. In this paper, we define the parameter 'points of influence' as the cut-off where points or a range of TC/HDL values are significantly associated with tone or entropy. 
(A)

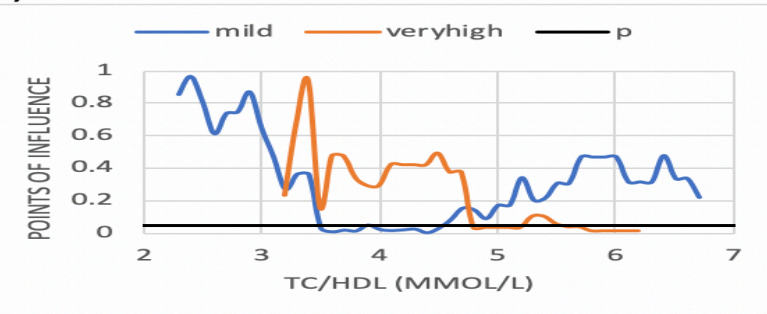

(B)

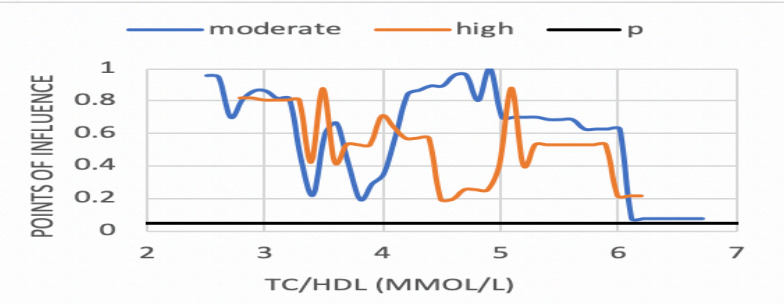

(C)

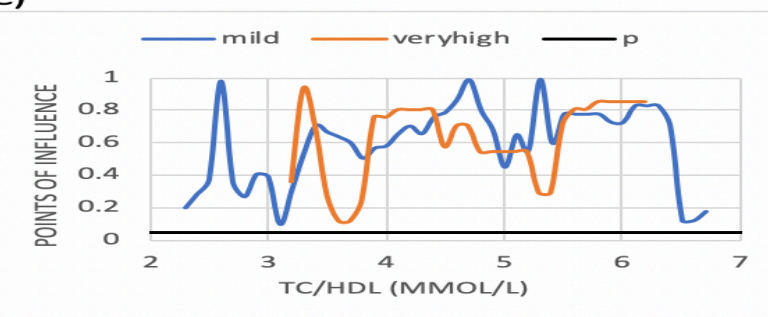

(D)

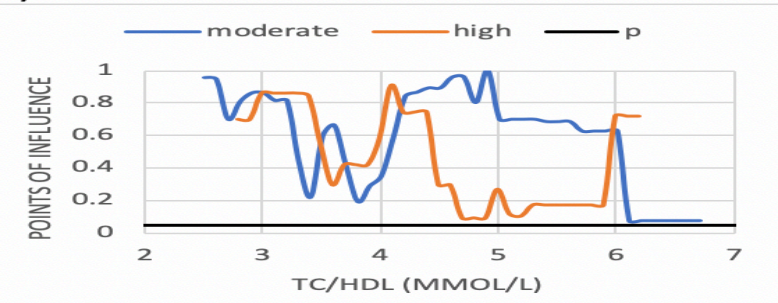

Figure 1: (A) Relationship between TC/HDL and tone for mild and very high Framingham CVD risk groups; (B) Relationship between TC/HDL and tone for moderate and high Framingham CVD risk groups. $\mathrm{p}$ - cut-off for significant association between tone and TC/HDL ; (C) Relationship between TC/HDL and entropy for mild and very high Framingham CVD risk groups; (D) Relationship between TC/HDL and entropy for moderate and high Framingham CVD risk groups. $\mathrm{p}$ - cut-off for significant association between entropy and TC/HDL

Table2. Cholesterol profile

\begin{tabular}{llllll}
\hline & Mild & Moderate & High & Very high & P \\
\hline $\begin{array}{l}\text { TC } \\
(\mathrm{mmol} / \mathrm{L})\end{array}$ & $5.20 \pm 1.0$ & $5.11 \pm 1.2$ & $4.64 \pm 0.9$ & $4.93 \pm 1.2$ & 0.0645 \\
$\begin{array}{l}\text { Trigs } \\
(\mathrm{mmol} / \mathrm{L})\end{array}$ & $2.36 \pm 13.3$ & $1.53 \pm 0.8$ & $1.56 \pm 0.9$ & $1.60 \pm 0.9$ & 0.953 \\
$\begin{array}{l}\text { HDL-C } \\
(\mathrm{mmol} / \mathrm{L})\end{array}$ & $1.42 \pm 0.4$ & $1.37 \pm 0.3$ & $1.28 \pm 0.4$ & $1.18 \pm 0.3^{*}$ & 0.0163 \\
$\begin{array}{l}\text { LDL-C } \\
(\mathrm{mmol} / \mathrm{L})\end{array}$ & $3.22 \pm 0.9$ & $3.04 \pm 1.0$ & $2.81 \pm 1.1$ & $3.06 \pm 1.1$ & 0.186 \\
TC/HDL & $3.83 \pm 1.1$ & $3.86 \pm 1.1$ & $3.89 \pm 3.89$ & $4.31 \pm 1.3$ & 0.271 \\
\hline
\end{tabular}

$\overline{\mathrm{DM}}$ - diabetes mellitus, TC - total cholesterol, Trigs - triglycerides, HDL-C - high density lipoprotein cholesterol, LDL-C - low density lipoprotein cholesterol, TC/HDL - total cholesterol, High density lipoprotein cholesterol ratio, ${ }^{*} \mathrm{p}<0.01$ for Mild vs. Very high.

Tone was shown to be significantly associated with TC/HDL for mild and very high risk groups (Figure $1 \mathrm{~A}$ ) but not in moderate and high risk groups (Figure $1 \mathrm{~B}$ ). The $\mathrm{P}$ value was significant $(\mathrm{P}<0.05)$ in the range 3.5 to 4.6 for mild risk group, and in the ranges between 4.8 to 5.2 and 5.6 to 6.1 for very high risk group. For entropy, no significant association was noted for any CVD risk group (Figure 1C,D).

Similarly, tone was significantly related to QTD length in mild and very high risk groups in the range between 38 to $62 \mathrm{msec}$ for mild risk group $(\mathrm{P}<0.05)$ and the ranges between 107 to $116 \mathrm{msec}$ and between 123 to $160 \mathrm{msec}$ for very high risk group $(\mathrm{P}<0.05)$ (Figure $2 \mathrm{~A}$ ). No significant association between entropy and QTD for mild and very high risk group (Figure $2 \mathrm{~B}$ ).

\section{Discussion}

Determination of final Framingham CVD risk score is based on blood analysis, which is an invasive procedure.
Simpler method that are non-invasive may correlate with

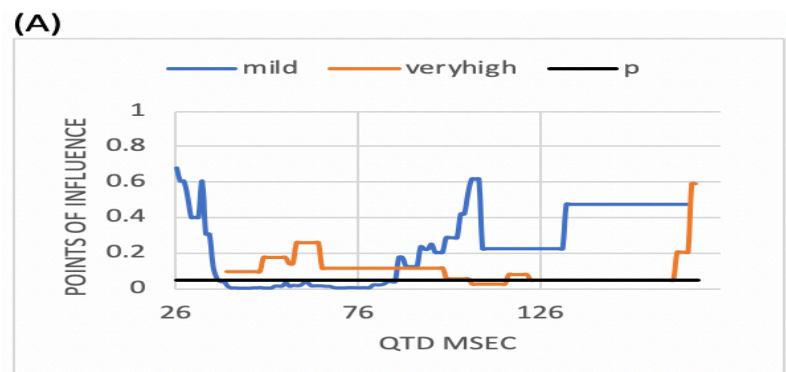

(B)

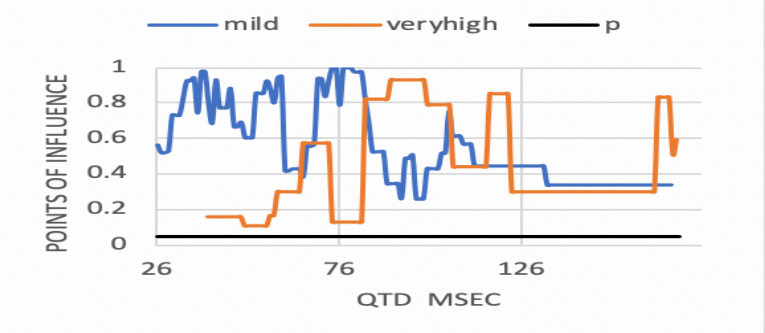

FIGURE II: (A) Relationship between QTD and tone for mild and very high Framingham CVD risk groups. $\mathrm{p}$ - cut-off for significant association between tone and QTD (B) Relationship between QTD and entropy for mild and very high Framingham CVD risk groups. p - cut-off for significant association between entropy and QTD

FRS and provides information in CVD risk. Our study focused on whether the CVD risk magnitude, as defined by FRS, is correlated with TC/HDL and QTD values by T-E algorithm. Our results indicated that tone is related to TC/HDL for mild and very high risk group. In previous work, tone has been shown to be associated to CAN, suggesting that it is the acceleration and inhibition of the heart rate measured by tone that is the main factor 
characterising any pathophysiology rather than entropy, which is a measure of the sympathovagal balance [13]. This might be why there was no direct relation between TC/HDL and entropy for any risk group.

There is a tight relation between TC/HDL and tone for normal intra-beat variation in the range between 3.8 to 4.9 [14]. This supports our finding with the mild risk group who have very similar intra-beat variation to normal individuals. However, the relationship between TC/HDL and tone was lost for the moderate and high CVD risk groups suggesting that the association between the autonomic nervous system network and blood biochemical network was dissipated. This loss in correlation between the two networks is due to the free radicals that damage the nervous system and blood vessel lining. Moreover, our results for tone analysis for very high risk group showed an association between tone and TC/HDL at increased level of $\mathrm{TC} / \mathrm{HDL}$ indicating that high risk of cardiovascular diseases dissociate the two networks, and thus increase the risk of sudden cardiac death due to CAN and arrhythmias in such population of high risk of cardiovascular diseases.

QTD measure the intra-beat variation which occurs in the electrical events of a single heart beat duration, whereas tone and entropy measure the inter-beat variation which occurs between two consecutive beats. Both variations are influenced by the parasympathetic and sympathetic of nervous system [15]. Our results for tone and QTD analysis showed an association between tone and QTD for mild risk group within a very similar range to normal subject which is in between 20 to $50 \mathrm{msec}$. However, for high risk group, the association between QTD and tone was at increased QTD. Previous study showed that increased QTD predicts arrhythmia and sudden cardiac death [16]. Our results are in line with these findings and extend them to high risk CVD population.

In this preliminary study, we conclude that tone is associated to both TC/HDL and QTD length, and is able to stratify mild and very high Framingham CVD risk groups. The advantage of this is that an easy to preform, noninvasive method can be utilized to identify subjects with mild and highly increased CVD risk.

\section{Acknowledgements}

Authors would like to thank Bev deJong for technical assistance. Roche Australia $\mathrm{P} / \mathrm{L}$ for providing the glucose test strips. This study was supported by Healthcare Engineering Innovation Center (HEIC) at Khalifa University.

\section{References}

1. Fox CS, Pencina MJ, Wilson PWF, Paynter NP, Vasan RS, D'Agostino RB. Lifetime risk of cardiovascular disease among individuals with and without diabetes stratified by obesity status in the Framingham Heart
Study. Diab Care. 2008;31(8):1582-4.

2. Deedwania PC. Silent myocardial ischaemia in the elderly. Drugs Aging. 2000;16(5):381-9.

3. Stocker R, Keaney JF, Jr. Role of Oxidative Modifications in Atherosclerosis. Physiol Rev. 2004 October 1, 2004;84(4):1381-1478.

4. Stocker R, Keaney JF, Jr. Role of Oxidative Modifications in Atherosclerosis. Physiol Rev. 2004;84(4):1381-478.

5. Ziegler D, Sohr CGH, Nourooz-Zadeh J. Oxidative Stress and Antioxidant Defense in Relation to the Severity of Diabetic Polyneuropathy and Cardiovascular Autonomic Neuropathy. Diab Care. 2004;27(9):2178-83.

6. Castelli W, Abbott R, McNamara P. Summary estimates of cholesterol used to predict coronary heart disease. Circulation. 1983;67(4):730-4.

7. Claudia C, Gil S, Katia B, Waldemar D, Aristarco Gongalves S-F. Clinical determinants of increased QT dispersion in patients with diabetes mellitus. Int J Cardiol. 2001 07/01;79(2):253-62.

8. Rosen MR, Jeck CD, Steinberg SF. Autonomic modulationof cellular repolarization and electrocardiographic QT interval. J Cardiovasc Electrophysiology. 1992;3:487-99.

9. Khandoker AH, Jelinek HF, Moritani T, Palaniswami M. Association of cardiac autonomic neuropathy with alteration of sympatho-vagal balance through heart rate variability analysis. Med Eng Phys. 2010;32(2):161-7.

10. Jelinek HF, Md Imam H, Al-Aubaidy H, Khandoker AH. Association of cardiovascular risk using non-linear heart rate variability measures with the framingham risk score in a rural population. Front Physiol. 2013;4:186.

11. Alexander R. W. (1995). Hypertension and the pathogenesis of atherosclerosis: oxidative stress and the mediation of arterial inflammatory response: a new perspective. Hypertension. 1995;25:155-161

12. Wilson P. W. F., D'Agostino R. B., Levy D., Belanger A. M., Silbershatz H., Kannel W. B. Prediction of coronary heart disease using risk factor categories. Circulation. 1998;97; 1837-1847

13. Khandoker AH, Jelinek HF, Moritani T, Palaniswami M. Association of cardiac autonomic neuropathy with alteration of sympatho-vagal balance through heart rate variability analysis Med Eng Phys. 2010;32:161-7.

14. Arumugam TV, Gleichmann M, Tang S-C, Mattson MP. Hormesis/preconditioning mechanisms, the nervous system and aging. Age Res Rev. 2006;5(2):165-78.

15. Lazar J, Manzella S, Moonjelly J, Wirkowski E, Cohen TJ. The prognostic value of QT dispersion in patients presenting with acute neurological events. J InvasCardiol. 2003;15(1):www.invasivecardiology.com/article/1232.

16. Stoletniy LN, Pai SM, Platt ML, Torres VI, Pai RG. QT dispersion as a noninvasive predictor of inducible ventricular tachycardia. J Electrocardiol. 1999;32(2):1737.

Address for correspondence:

Ahsan Khandoker

Healthcare Engineering Innovation Center (HEIC)

Department of Biomedical Engineering

Khalifa University of Science and Technology

Main Campus, Abu Dhabi PO Box 127788 UAE 